\title{
The Autonomous Miniature Robot Alice: from Prototypes to Applications.
}

\author{
G. Caprari, K. O. Arras, R. Siegwart \\ Swiss Federal Institute of Technology Lausanne (EPFL) \\ Institute of Robotic Systems \\ EPFL, DMT-ISR, CH-1015 Lausanne \\ E-mail: Gilles.Caprari@epfl.ch \\ http://dmtwww.epfl.ch/isr/asl
}

\begin{abstract}
For any commercial product, design cycles from the idea, to concept, prototype and test are iterative processes. This is especially true for high technology products. In the last years we acquired experience in designing and re-designing the small mobile robot Alice. In the first part of this paper we present an overview of the prototype family of Alice and the improvements achieved so far. Applications are often the final objective but also an incentive to correct and enhance the robot abilities. In the second part, research carried out with Alice and various real-world applications, which exceed the robot's use as a research prototype, will be presented. They include local and global localization, map building, control strategies for semi-autonomous operation via Internet and Matlab, its use for robot soccer tournaments and as a research platform for studies of collective behaviors, and finally, ludic applications.
\end{abstract}

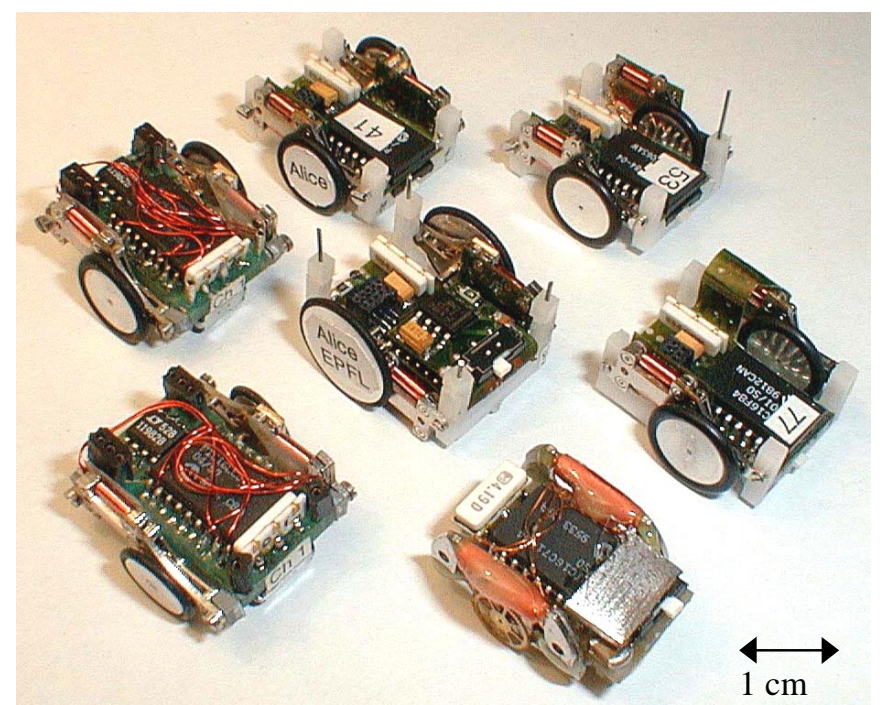

Figure 1. Evolution of prototypes

\section{Introduction}

Mobile robotics is a relatively new research field. Whereas stationary manipulators are widely used in industry, mobile robots have still difficulties to find real applications. Often it is preferable to modify the environment and to use very limited automated vehicles than invest in more autonomous mobile robots able to deal with a complex world. For mini mobile robots (MMR) it is even more difficult to find applications and realize them. However they are still of high interest and one can imagine plenty of applications at long term. Inspection of installations difficult to reach by humans has high economic interest and will be investigated in future. Exploration of hazard environments or distant places has been studied an implemented with small rover. These rovers are becoming small and smaller but they are still quite big compared to existing laboratory mini robots.

While waiting for a future where MMRs will probably be workers in micro factories, transporting micro parts from one assembly line to the other, we will use these micro robots in more realistic scenarios. Applications can be found as research and education tool, as demonstrators in expositions, for inspections tasks, for experiments in the field of multirobot collective behavior and in the toy market.

This paper is organized in three major sections. First we enumerate the successive evolution of the robot hardware which in the meantime spans five years. In the second section we present on-going research carried out on the robot. Finally, several real-world applications of the robot are presented. There are many relations between these sections since hardware improvements were undertaken for certain applications but also when new electronics components became available or novel applications were suggested.

\section{History of development}

Figure 1 shows the evolution of the Alice base module over five years. The oldest one is in the bottom right of the picture, progressing clockwise until number 77 . In the center we find the latest version: Alice 99.

\subsection{Predecessor of Alice}

The first robot in figure 1 was built at the ETHZ (Swiss Federal Institute of Technology Zurich) in 1995 [Büchi95]. Components are:

- 2 monodirectional watch motors 
- microcontroller PIC16C71

- 1 lithium battery (3V)

- 1 LED and 2 phototransistors

- oscillator, power switch and PCB

This robot is able to follow a black line on a white paper. The goal was to demonstrate the combination of watch motors with low power microcontroller in an autonomous integrated system. This early robot worked well but had several drawbacks. As the wheels are directly the seconds' gears, the robot slips on smooth surfaces. The motors had to be disassembled and modified and thus the robot is difficult to assemble. Finally the microcontroller can be programmed only once such that no changes on the control algorithm are possible.

\subsection{Alice 697}

This version has also been developed at the IfR (Institute of Robotics) at ETHZ [Büchi96]. The objectives were to build the mini robot as flexible as possible, able to sense obstacles and receive wireless commands. Software flexibility is assured by the re-programmable EEPROM microcontroller, while hardware flexibility is obtained with extension connectors for additional sensor modules. A module with 2 proximity sensors and an Infrared receiver extends the basic functionality of this prototype. The components on the main module are:

- microcontroller PIC16C84

- AD converter 2 channels

- extension connectors

- 2 bidirectional watch motors

- 2 aluminum wheels with rubber tire

- 2 silver-oxide battery (1.55V)

- batteries holder

- oscillator, power switch

From this version on, we started to use bidirectional watch motors with wheels mounted on the minute hand. This extends the mobility of the vehicle and the rubber tires assure enough grip on any surface.

\subsection{Alice ‘98}

For this version, developed at the Swiss Federal Institute of Technology Lausanne (EPFL), a big effort towards simplification of assembly was made [Caprari98]. Any loose wires to the motors have been avoided and all components were put onto a flexible print. The new plastic frame and the bigger rubber tires absorb part of the shocks and thus augment the mechanical robustness of the robot. From this version on, particular attention was paid to reduce costs, permitting multi robot approaches and bigger markets. With a smaller connector and a serial bus concept we opened the way for more sophisticated extensions modules like a fourdirection proximity sensor module and bidirectional radio communication. The base module contains all the essential components assuring the mobility:

- 2 bidirectional watch motors
- 2 aluminum wheels with rubber tire

- microcontroller PIC16C84

- 2 silver-oxide battery $(1.55 \mathrm{~V})$

- extension connector

- plastic frame for support and batteries holder

- flexible PCB connecting also the motors

- oscillator, power switch and capacitor

This version of Alice won the International Nagoya Maze Contest 1998 in the category for autonomous systems.

\subsection{Alice '99}

The most current version of 1999 is now commercialized by K-Team, a spin-off company of EPFL. New features are bigger wheels, now symmetrically mounted on a common axis, and a regulated power supply. In the previous versions, the wheels were placed in an asymmetric way due to the shape of the motor. This yields slipping as soon as the robot turns, leading to poor odometric pose estimates and demanding higher torque in a curve. The new solution with three batteries and a voltage regulator stabilize the power supply and thus additional modules have enough power to work properly. The problem derived from the high internal resistance of the small coin batteries. When the system required some additional milliamps, the voltage decreased causing malfunctions to the motors and other electronic. For modules like the bidirectional radio, this resulted in communications errors of a non-negligible extent. With this new version, the base module delivers stable voltage until 10 $\mathrm{mA}$. For such small systems power management remains a primary issue to deal with from hardware up to software. On the latest base module we find:

- 2 bidirectional watch motors

- 2 symmetric wheels

- microcontroller PIC16F84

- 3 silver-oxide battery $(1.55 \mathrm{~V})$

- voltage regulator

- extension connector

- plastic frame for support and batteries holder

- flexible PCB connecting all the components

- oscillator, power switch and capacitor

There are various extension modules that can be connected to the base:

- 4 IR proximity sensor module

- IR remote controller receiver module

- 2 IR proximity sensors and IR receiver module

- standard TV remote receiver module

- bidirectional radio communication module

This version won for the second time the International Nagoya Maze Contest 1999 in the category of autonomous systems.

Totally more than hundred Alices were built up to now, particularly from the two last versions because of the reduced cost and assembly time. About eighty Alices are now on duty in several laboratory and exhibitions. 


\section{Research}

\subsection{Navigation}

MMRs are obvious candidates for remote inspection tasks. Man-made infrastructures where small structural dimensions stand in contrast to a large overall size of the entire system motivate the application of autonomous micro systems for inspection and on-site manipulation. Examples include building infrastructure like ventilation systems or small diameter pipeline systems. Hardware requirements for such missions are mobility, high autonomy with respect to energy, embarked sensors and communication, and high mechanical and electrical robustness and reliability. On the software side the robot must exhibit partial autonomy for reactive local navigation maneuvers, should be able to navigate globally and ask for help in situations which are beyond its capacities. Similar requirements must be satisfied for remote exploration in space robotics [Wilcox96]. These type of missions, for robots in the size of Alice, are an on-going research subject and still remain to be realized. However the building blocks and tools for mobile micro-robots in the abovementioned application scenarios can be identified as: local and global localization, map-building, distributed control algorithms and user interface.

\subsubsection{Limitations and approach}

The sensors which are available and practicable for MMRs such as Alice impose severe limitations when reliable information for navigation is required. This is valid for interoperceptive sensors like odometry and particularly for exteroperceptive sensors like range or intensity finders. In contrast to big mobile robots, non-systematic odometry errors stemming from uneven floors, wheel slippage or external forces appear in the same order of magnitude as systematic errors. This in combination with unreliable exteroperceptive sensors makes navigation of MMRs a particularly difficult task. On the other hand, most of the abovementioned environments are man-made and very structured.

The kind of sensory information which is available for MMRs makes metric navigation difficult. Metric navigation explicitly represents and estimates the vehicle position and orientation in a global or local reference frame. It relies typically on precise sensory information and good models for sensors and actuators. A topology-based approach for navigation of MMRs appears to be more appropriate since it is less model-based and maintains qualitative information without the need for high precision. Being able to recognize locally unique places, and equipped with a library of simple motion behaviors, the robot can navigate in a known environment between these places which serve as points for localization [Kuipers, Byun 89].
Starting from a remote inspection scenario, structured, labyrinth-like environments have been used as test bed for Alice's navigation algorithms.

\subsubsection{Local and global localization}

Localization is a basic skill for a mobile robot which is supposed to navigate globally in an autonomous mode. With local localization we refer to robot pose estimation in known environment when the previous pose is approximately known. Global localization is the task of finding the robot pose in known environment without a priori knowledge on the pose (e.g. robot is lost). For both localization types, Alice extracts four different topological primitives which are typical for our environment type: single connection situation (dead-end, referred to as $I$ ), two connection situation (rightand left-sided $L$ ), three connection situation ( $T$-crossing) and four connection situation ( $X$-crossing). The extraction algorithm searches for statistically accumulated evidence on jumps in the raw sensor readings. Each primitive exhibits a characteristic combination or succession of jumps. These locations define the locally unique places which serve as points for localization. For place-to-place navigation the robots possesses three behaviors: obstacle avoidance, wallfollowing left and wall following right. Equipped with these elements, the robot is able to localize itself each time when it traverses a distinctive place (figure 2).
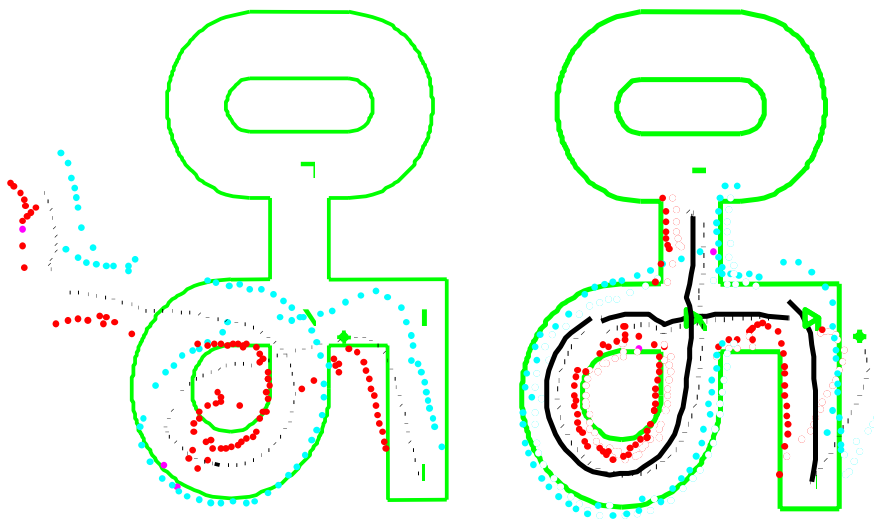

Figure 2. The effect of localization: without (left) and with (right). The points in light gray (blue) are raw range readings from the right sensor, points in dark gray (red) from the left sensor. The trace at the right displays the corrected robot trajectory. Jumps in the trace depict the localization corrections.

For global localization, the robot navigates from its start point randomly with the obstacle avoidance behavior. The places it traverses and recognizes are stored forming a sequence of symbols from the alphabet $\{I, L, T, X\}$. A search algorithm then tries to match the symbol sequence in the a priori known map. Multiple position hypotheses are maintained. As soon as the sequence becomes globally unique, the robot is re-localized. The matching algorithm allows wildcard symbols in the sequence as well. This is of great importance since false place detection can occur. In 
such a case, the matching stage is able to eliminate symbol sequences which are impossible in the environment. Thus, false detections can not only be recognized but also autocorrected, yielding a high degree of robustness for localization. The corresponding Matlab interfaces are shown in figures 3 and 4.

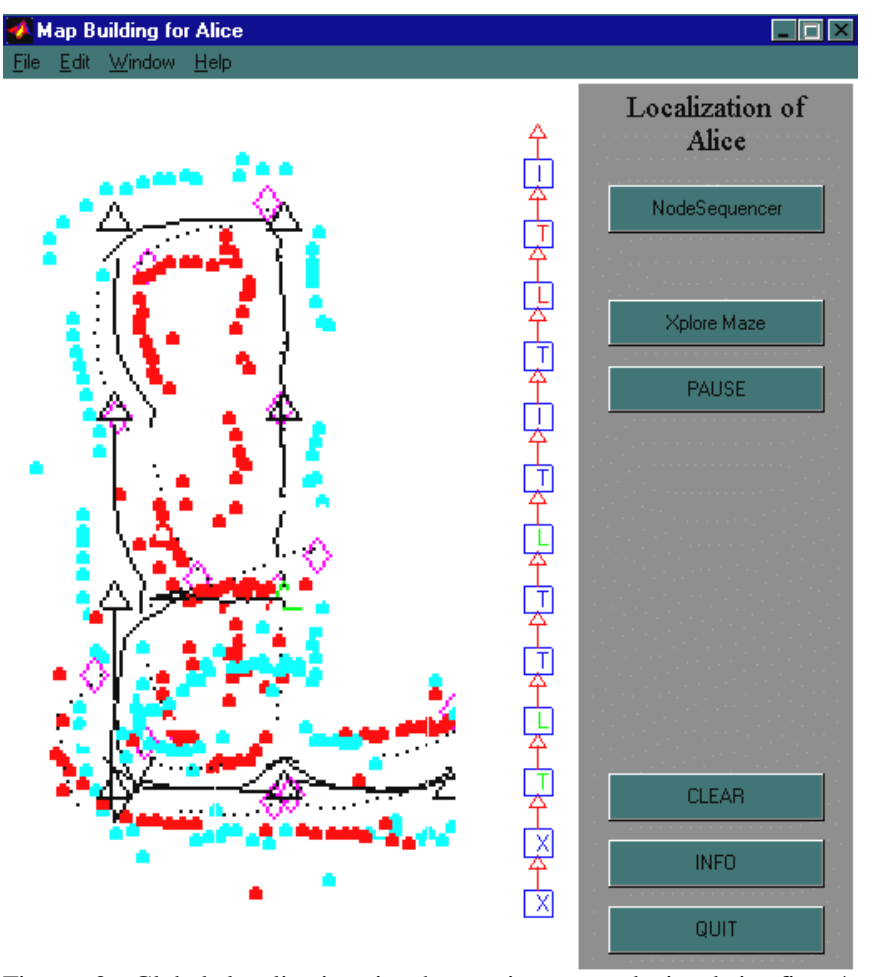

Figure 3. Global localization in the environment depicted in fig. 4. Corrected recognizable locations are marked by a triangle. The robot started from an unknown position. The vertical chain shows the sequence of the last thirteen detected places.

\subsubsection{Map-building}

Map building with MMRs is a challenge since odometry provides only pose estimates of very low quality. However experiments with topological navigation supported also by rough metric information have been conducted. The robot is able to detect openings with a similar algorithm as for the extraction of topological primitives. Unexplored openings are stored on a stack and processed with a backtracking technique. Exploration is finished if all open connections have been examined. In addition to this, metric has to be introduced in order to recognize already visited places. This is necessary because the locally distinctive places are not globally unique as there are several identical $I, L, T, X$ in the environment. For the same purpose, the assumption of an orthogonal environment structure has been made. This assumption denotes a limitation to a certain environment type but is still compatible with the application scenarios we consider. Applying this assumption, odometry can be corrected to an uncritical extent allowing distinguishing places and also transforming raw data into the global frame with relative high precision. The resulting maps contain the metric position of the raw data and a graph-like representation of the environment topology.

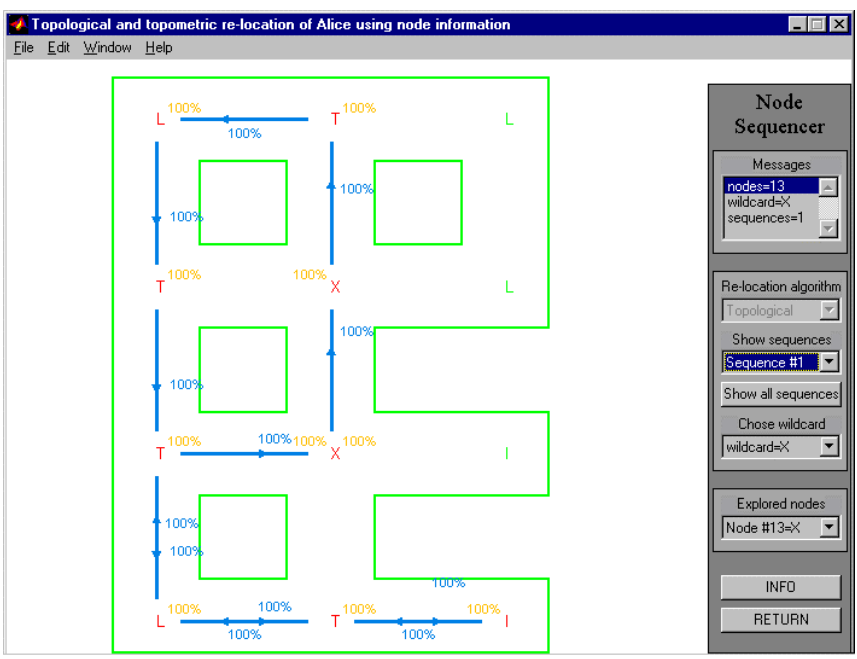

Figure 4. The environment and the path found for the symbol sequence gained during navigation of figure 3 . Starting from an unknown position, the robot is successfully localized.

\subsection{User interface design}

Figures 3 and 4 show the user interface to Alice for all kind of navigation tasks. It has been implemented in MATLAB and satisfies modern guidelines of ergonomic interface design. The interface provides means for task specification and supervision. High-level and low-level navigation commands can be sent to the robot. All off-board calculations are done in MATLAB as well.

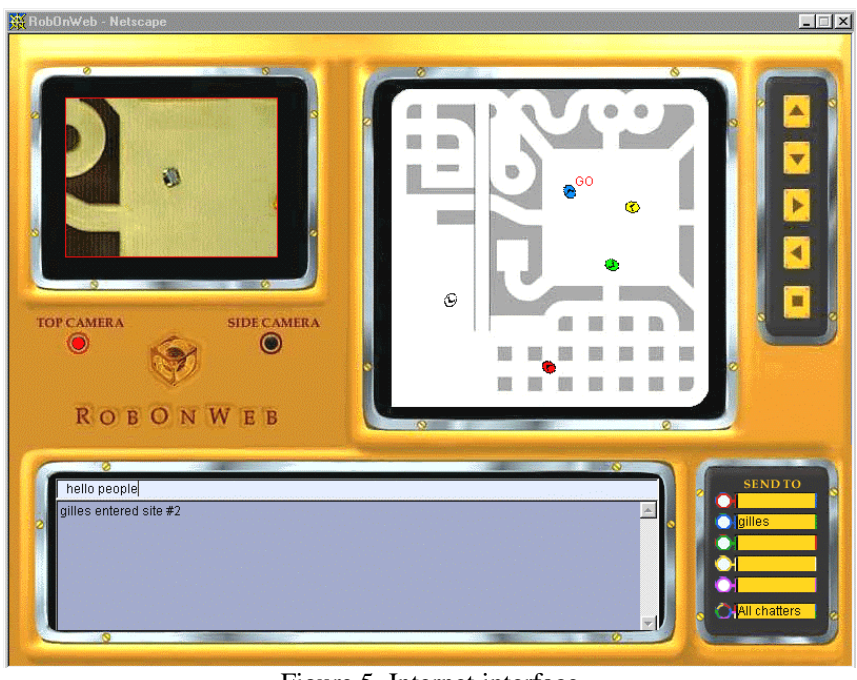

Figure 5. Internet interface

Another kind of interface (figure 5) was chosen for teleoperation [Siegwart98]. Information is transferred via Internet and displayed on a web page (RobOnWeb.Verkehrshaus.org). Only a small real image around the area of interest is available on the user interface because of the limited transfer rate. A map with updated 
robot positions extends the information available on the web interface. The third window permits to chat between users. To our knowledge, this is the only Internet set-up featuring multiple robots, and thus we want the users to communicate and cooperate for common tasks.

\subsection{Studies in multi-robot collective behaviors}

A research field of high interest concerning MMRs are bioinspired approaches towards complex systems dynamics and collective behavior. When we deal with small units with limited intelligence the analogy with e.g. social insects is evident. The relation between the single agents acting and inter-acting on a local level, and thus contributing to the emerging overall intelligence of a society of agents is one of the issues of interest. Developing a MMR for such experiments is a challenge for roboticists since the users are often not technicians (e.g. biologist) and therefore, simple tools for programming the robot have to be provided. From the biology point of view the interest is to better understand insects and their controlling law, testing and verifying new models [Bonabeau99]. Understanding the underlying methods to control swarms of decentralized minirobots is also of interest for robotic applications. Scenarios with a multitude of cooperating MMRs, exploring or inspecting partially known environments, ask for new control strategies, which could be gained from biology or from ideas obtained by an interdisciplinary approach of robotics and biology.

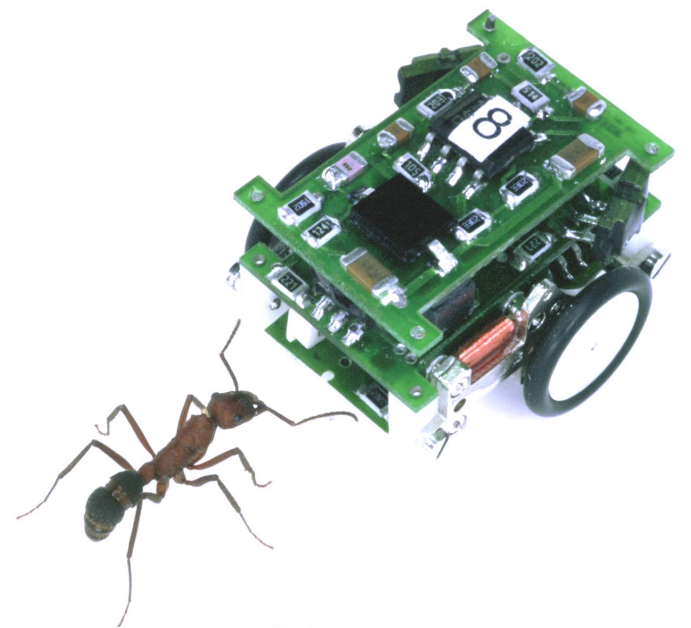

Figure 6. Alice with a study mate

\section{Application}

\subsection{Exhibitions}

Exhibitions and performance demonstrators give the possibility to present mobile robotics in general to a large public. We believe that in future, mobile robots will play an important role in the every day's life and will help humans in their environment or will just play with them. Presenting families of non-dangerous small robots promote the idea of the robot as an every day's companion.

A newer promising trend is to use the fascination of small moving machines to catch the attention of a visitor during fairs or museums. The advertisement market is surely interested by this solution.

We presented Alice in many exhibitions and we had a vast success. At the Swiss Museum of Transport we installed a permanent set-up with five Alices attainable via Internet (www.RobOnWeb.Verkehrshaus.org). It is to notice that the set-up and the robots just need a simple maintenance from an unspecialized technician. One year of interrupted operation is the minimal prerequisite for such installation.

\subsection{For the public as a demo and a toy}

Always related to the attraction created by nice small moving intelligent vehicles, it is possible to attack the toy and entertainment market. The benchmark for the roboticist is to build a robust, cheap, but complex and integrated system. We envisage a high-level line, ranging from the hobbyist to more exclusive toys. This still permits hi-tech relative expensive implementation and more intelligent products. In the near future also low cost simple toys will be of interest. The fact of attacking a broad market like this, permits to augment the number of produced pieces, develop expensive tools and parts, amortize the initial costs and reduce the final cost. This is an advantage also for the research, especially for collective approaches. Alice is now also a commercial product and many people, ranging from children to professors, expressed their interest. For people who just want to play with the robot as a tele-operated intelligent minicar, we implemented remote control via a standard TV infrared remote control unit (figure 7).

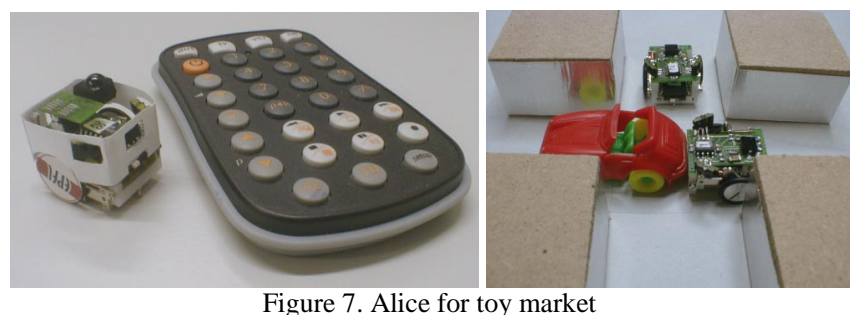

\subsection{Education}

As incentive for students, the maximum they can get are small moving robots following their control program. A benchmark is necessary, and surely the most known game is soccer. Besides being well known (the game and the rules), soccer can be played with an evolving complexity with virtually no limits and it is open to collective/cooperative solutions. Often the organized competitions comprehend not only the game itself but also the construction of a robot kit, an overall vision system and a simulator. The complexity and the interdisciplinarity may discourage some students. Presented solutions often work not well or are good just in 
software or mechanically. If we want the abstraction level to be higher, we have to supply the competing teams with good tools and partially working systems. For this reason we developed a set-up to play soccer on an A4 $(21 \times 30 \mathrm{~cm})$ field. The system is composed by:

- 2 teams of 3 Alice each

- Vision system (USB Camera)

- Controlling system and interface on a PC

- An infrared remote controller

- The field

The included PC is in charge of tracking the robot position via image processing, sending command to the 6 robots via IR and interfacing the 2 teams via Ethernet. The user connects to the system via network and using a defined protocol, receives the robot positions and send the control messages. It is possible to interact at different level: speed control, position control and behavior control. This corresponds to an increasing abstraction and a decreasing complexity. The expert user will control directly each wheel speed, whereas the novice will take advantage of a set of existing robot behaviors.

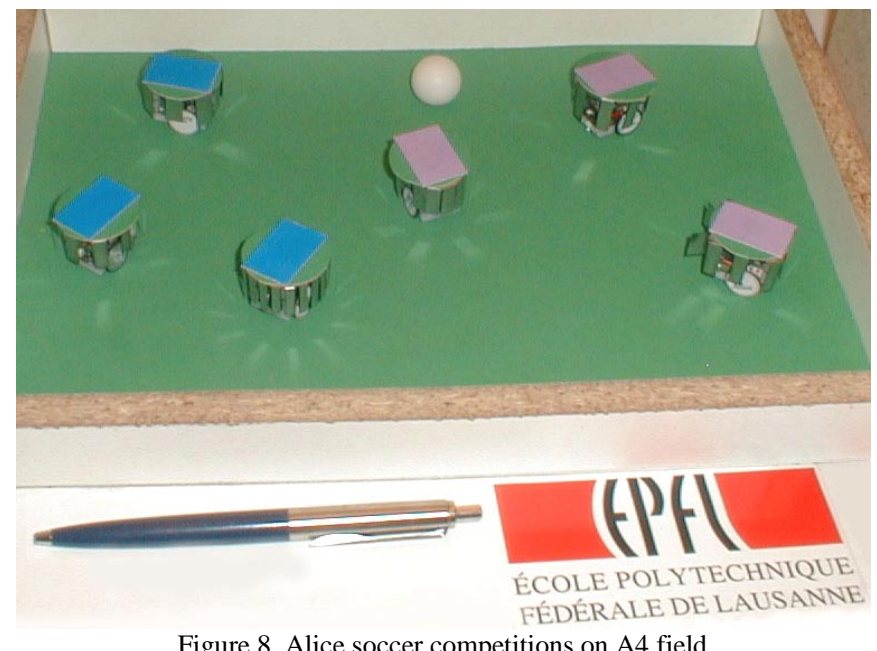

\section{Conclusion}

In this paper we presented three aspects of the MMR Alice: the development, research and the application. We presented the hardware evolution as being strongly related to the requirements from the two other domains, leading to iterative design and re-design of the mechanical, electrical and software concept.

As many problems in miniature robotics are still to be solved and thus real-world applications are still far away, the robot in its actual version is in daily use in several research and exhibition applications, providing a high degree of reliability and requiring a minimum of maintenance. Approximately hundred units of this robot have already been built. It is also commercially available.

Further effort is put on system optimization towards increased mechanical robustness, lower production cost and enhanced autonomy. Going in this direction will surely make possible a much wider use of intelligent mobile mini robots in fields even not imaginable at this point.

\section{References}

[Büchi95] R. Büchi, D. Rohrer, C. Schmid, R. Siegwart, “A fully Autonomous Mobile Mini Robot" SPIE Photonics EAST '95, Philadelphia, pp. 50-3, 1995

[Büchi96] R. Büchi, G. Caprari, V. Vuskovic, R. Siegwart, "A remote controlled mobile mini robot", Int. Symposium on Micro Machine and Human Science, pp 203-6, 1996.

[Caprari98] G. Caprari, P. Balmer, R. Piguet, R. Siegwart, "The Autonomous Micro Robot ALICE: A platform for Scientific and Commercial Applications", Int. Symposium on Micromechatronics and Human Science, pp 231-5, 1998.

[Bonabeau99] E. Bonabeau, M. Dorigo, G. Theraulaz, "Swarm Intelligence: From Natural to Artificial Systems", Oxford University Press, July 1999.

[Wilcox96] B. Wilcox et al, "Nanorovers for Planetary Exploration" AIAA Robotics Technology Forum, Madison, WI, August 1996, pp. 11-1-11-6.

[Siegwart98] R. Siegwart, C. Wannaz, P. Garcia, R. Blank, "Guiding Mobile Robots through the Web", Workshop Proc. of IEEE/RSJ International Conference on Intelligent Robots and Systems, Victoria, Canada, October 12-16, 1998

[Kuipers, Byun 89] Kuipers B.J., Byun Y.T., “A Robust, Qualitative Approach to a Spatial Learning Mobile Robot", Proceedings of the SPIE, Sensor Fusion: Spatial Reasoning and Scene Interpretation, Vol. 1003, 1988. 